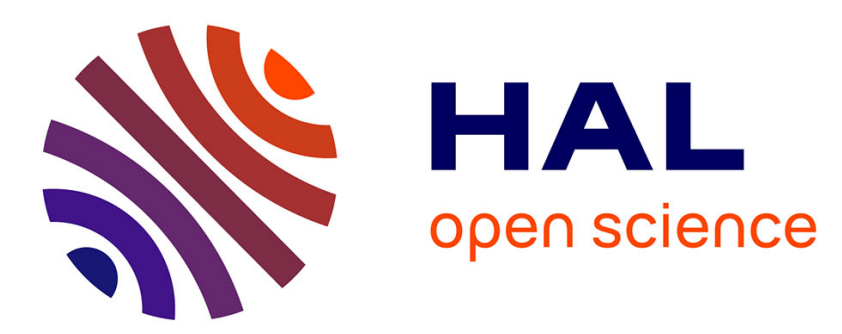

\title{
A New Tubular Structure Tracking Algorithm Based On Curvature-Penalized Perceptual Grouping
}

\author{
Li Liu, Da Chen, Minglei Shu, Huazhong Shu, Laurent D. Cohen
}

\section{To cite this version:}

Li Liu, Da Chen, Minglei Shu, Huazhong Shu, Laurent D. Cohen. A New Tubular Structure Tracking Algorithm Based On Curvature-Penalized Perceptual Grouping. IEEE International Conference on Acoustics, Speech and Signal Processing, ICASSP 2021, Jun 2021, Toronto, Canada. 10.1109/icassp39728.2021.9414114 . hal-03424096

\section{HAL Id: hal-03424096 https://hal.science/hal-03424096}

Submitted on 10 Nov 2021

HAL is a multi-disciplinary open access archive for the deposit and dissemination of scientific research documents, whether they are published or not. The documents may come from teaching and research institutions in France or abroad, or from public or private research centers.
L'archive ouverte pluridisciplinaire HAL, est destinée au dépôt et à la diffusion de documents scientifiques de niveau recherche, publiés ou non, émanant des établissements d'enseignement et de recherche français ou étrangers, des laboratoires publics ou privés. 


\title{
A NEW TUBULAR STRUCTURE TRACKING ALGORITHM BASED ON CURVATURE-PENALIZED PERCEPTUAL GROUPING
}

\author{
Li Liu ${ }^{1} \quad$ DaChen ${ }^{2} \quad$ Minglei Shu $\quad$ Huazhong Shu $\quad$ Laurent D. Cohen ${ }^{4}$ \\ ${ }^{1}$ School of Computer Science and Technology, Donghua University, Shanghai, China \\ ${ }^{2}$ Shandong Artificial Intelligence Institute, Qilu University of Technology,Jinan,China \\ ${ }^{3}$ Laboratory of Science and Technology, Southeast University, Nanjing, China \\ ${ }^{4}$ University Paris Dauphine, PSL Research University, Paris, France
}

\begin{abstract}
In this paper, we propose a new minimal path-based framework for minimally interactive tubular structure tracking in conjunction with a perceptual grouping scheme. The minimal path models have shown great advantages in tubular structures tracing. However, they suffer from shortcuts or short branches combination problems especially in the case of tubular network with complicated structures or background. Thus, we utilize the curvature-penalized minimal paths and the prescribed tubular trajectories to seek the desired shortest path. The proposed approach benefits from the local smoothness prior on tubular structures and the global optimality of the graph-based path searching scheme. Experimental results on synthetic and real images prove that the proposed model indeed obtains outperformance to state-of-the-art minimal pathbased algorithms.
\end{abstract}

Index Terms - Tubular structure tracking, minimal path, perceptual grouping, curvature regularization

\section{INTRODUCTION}

Tubular structure tracking is an important task in many image analysis fields of computer vision and medical image analysis, a basic target for which is to search for the centerline or boundaries to delineate an elongated structure. The existing tubular structure tracking approaches can be roughly divided into automatic tracking models and interactive models.

A simple idea for automatic tubular structure tracking is implemented by growing curves iteratively from a set of seed points based on local tubular features [1,2]. Unfortunately, they may suffer from difficulty in coping with tubular structures with gaps. The implementation of minimal paths is an alternative solution for tracking a connected tubular

Thanks to the Shanghai Sailing Program (No.20YF1401500), the Fundamental Research Funds for the Central Universities (No.2232020D-35), the National Natural Science Foundation of China (No.61902224), and the French government under management of Agence Nationale de la Recherche as part of the "Investissements d'avenir" program, reference ANR-19-P3IA0001 (PRAIRIE 3IA Institute) for funding.
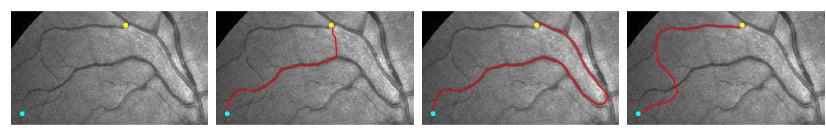

Fig. 1: Short branches combination problems. Columns1-4: Original image, Results from [7], [9] and the proposed method.

structure tree automatically. Significant examples include the keypoints-based models [3], the geodesic voting methods [4] and the minimum spanning tree models [5].The minimal path model is regarded as one of the most successful interactive tools in tracing tubular structures, by which a tubular structure can be naturally treated as a minimal path. The original minimal path model [6] provides a Eikonal PDE framework for tubular structure tracing. However, there is no guarantee that the minimal paths pass through the exact tubular centerlines. Significant improvements on tracing the tubular structure have been made by designing different metrics. An abstract dimension representing the thickness of tubular structures is added to track the centerlines and boundaries simultaneously [7]. The dynamic metric models [8] incorporate the update procedure of geodesic metrics during the fronts propagation. The curvature regularization is introduced to minimal path computation in either continuous domain [9] or discrete domain [10].

Despite the above efforts, the short branches combination problem still occurs when dealing with complicated situation, as depicted in Figs. 1.2 and 1.3. In this paper, we propose a new tubular structure tracing model which combines the curvature-penalization geodesic distance and prescribed trajectories. The curvature-penalized geodesic path is introduced to recover the gap between two neighbouring trajactories, which is more accurate and natural.

\section{BACKGROUND ON CURVATURE-PENALIZED MINIMAL PATH MODEL}

The curvature-penalized minimal paths $[11,12,9]$ utilize the curvature property instead of the curve length [6], as a reg- 

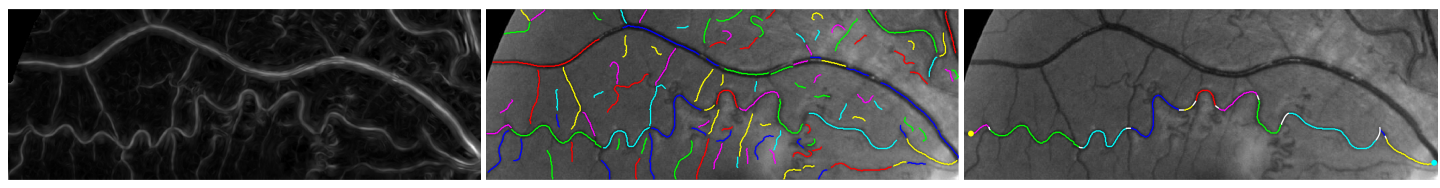

Fig. 2: The procedure of the proposed tubular structure tracing model. 1 Visualization for the vessel score map $\psi$. 2 Set of trajectories superimposed on the image. 3 The final path obtained by connecting the gaps between adjacent trajectories using bridging paths (white lines)

ularization term, which yields minimal paths with strongly smooth and elastic property.

Denote by $\tilde{\Omega}=\Omega \times \mathbb{S}^{1}$ an orientation-lifted space, where $\Omega \subset \mathbb{R}^{2}$ is an open and bounded image domain and $\mathbb{S}^{1}=$ $[0,2 \pi]$ is an interval with periodic boundary condition. Basically, the curvature-penalized minimal path models aim to minimize a curve length functional measured along a regular curve $\gamma:[0,1] \rightarrow \tilde{\Omega}$ with second-order derivative as follows:

$$
\mathcal{L}(\gamma)=\int_{0}^{1} \mathcal{F}\left(\gamma(u), \gamma^{\prime}(u)\right) d u
$$

where $\mathcal{F}: \tilde{\Omega} \times \mathbb{R}^{3} \rightarrow \mathbb{R}^{+}$is a geodesic metric over the tangent bundle of the manifold $M:=\tilde{\Omega}$. It implicitly encodes a curvature term. The geodesic distance map $\mathcal{U}_{\tilde{a}}$ very often lends itself to the minimization of the weighted curve length $\mathcal{L}$ :

$$
\mathcal{U}_{\tilde{a}}(\tilde{x}):=\inf _{\gamma}\{\mathcal{L}(\gamma), \gamma(0)=\tilde{a}, \gamma(1)=\tilde{x}\} .
$$

We exploit the the Finsler variant of the sub-Riemannian (FSR) model where the metric $\mathcal{F}:=\mathcal{F}_{\epsilon, \beta}$ can be formulated for any point $\tilde{x}=(x, \theta) \in \tilde{\Omega}$ and any vector $\tilde{\mathbf{u}}=(\mathbf{u}, \nu) \in \mathbb{R}^{3}$

$$
\mathcal{F}_{\epsilon, \beta}(\tilde{x}, \tilde{\mathbf{u}}):=\mathfrak{C}(\tilde{x}) \mathfrak{F}_{\epsilon, \beta}(\tilde{x}, \tilde{\mathbf{u}}),
$$

where $\epsilon \in[0,1]$ and $\beta \in \mathbb{R}^{+}$are penalty parameters, $\mathfrak{C}: \tilde{\Omega} \rightarrow$ $\mathbb{R}^{+}$is an orientation-dependent function derived from the image data and the vector $\mathbf{n}_{\theta}$ is defined as $\mathbf{n}_{\theta}=(\cos \theta, \sin \theta)$. The function $\mathfrak{F}_{\epsilon, \beta}$ is the FSR metric that can be expressed by

$$
\begin{aligned}
\mathfrak{F}_{\epsilon, \beta}^{2}(\tilde{x}, \tilde{\mathbf{u}})= & \left|\left\langle\mathbf{u}, \mathbf{n}_{\theta}\right\rangle\right|^{2}+\beta|\nu|^{2}+\epsilon^{-2}\left(\|\mathbf{u}\|^{2}-\left|\left\langle\mathbf{u}, \mathbf{n}_{\theta}\right\rangle\right|^{2}\right) \\
& +\left(\epsilon^{-2}-1\right) \min \left\{0,\left\langle\mathbf{u}, \mathbf{n}_{\theta}\right\rangle\right\}^{2}
\end{aligned}
$$

It is known that the geodesic distance map $\mathcal{U}_{\tilde{a}}$ satisfies the Eikonal equation such that $\mathcal{U}_{\tilde{a}}(\tilde{a})=0$ and for any orientationlifted point $\tilde{x} \in \tilde{\Omega} \backslash\{\tilde{a}\}$ we have

$$
\max _{\tilde{\mathbf{v}} \neq \mathbf{0}} \frac{\left\langle\nabla \mathcal{U}_{\tilde{a}}(\tilde{x}), \tilde{\mathbf{v}}\right\rangle}{\mathcal{F}_{\epsilon, \beta}(\tilde{x}, \tilde{\mathbf{v}})}=1 .
$$

The Eikonal equation (4) can be solved by using the stateof-the-art Finsler variant of the fast marching method [13]. A geodesic path $\mathcal{C}_{\tilde{a}, \tilde{x}}$ linking from $\tilde{a}$ to $\tilde{x}$ can be derived by re-parametering the solution $\mathcal{C}$ to a gradient descent ordinary differential equation (ODE) on the geodesic distance map $\mathcal{U}_{\tilde{a}}$

$$
\mathcal{C}^{\prime}(u)=\underset{\|\tilde{\mathbf{v}}\|=1}{\arg \max }\left\{\frac{\left\langle\tilde{\mathbf{v}}, \nabla \mathcal{U}_{\tilde{a}}(\mathcal{C}(u))\right\rangle}{\mathcal{F}_{\epsilon, \beta}\left(\tilde{\mathbf{v}}, \nabla \mathcal{U}_{\tilde{a}}(\mathcal{C}(u))\right)}\right\} .
$$

The metric $\mathfrak{F}_{\epsilon, \beta}$ implicitly encodes curvature penalization such that the FSR minimal paths are the approximate minimizers to the cost $\int_{0}^{1} \sqrt{1+\beta \kappa(u)^{2}} d u$ with in-reversible constraint on paths, where $\kappa:[0,1] \rightarrow \mathbb{R}$ is the curvature of $\gamma$.

\section{TRAJECTORIES GROUPING FOR TRACING TUBULAR STRUCTURES}

The tubular structure may cross over another one with stronger appearance, or lie in complicated background. The short branches combination or shortcuts problem often occur when exploiting minimal path approaches for tubular trajectory tracing. We propose a new minimal path model for minimal interactive tubular structure tracking, in conjunction with FSR geodesic distance estimation and Dijkstra's short path algorithm [14], as depicted in Fig. 2. The proposed model is built on a graph $\mathcal{G}=(\mathcal{V}, \mathcal{E})$, where $\mathcal{V}$ represents the node set and $\mathcal{E}$ stands for the edge set. $e_{i j} \in \mathcal{E}$ denotes the edge linking two nodes $\vartheta_{i}$ and $\vartheta_{j} \in \mathcal{V}$. Each edge $e_{i j}$ will be assigned a weight value $\omega_{i j} \in \mathbb{R}_{0}^{+}$and $\omega_{i j}=+\infty$ implies that the node $\vartheta_{i}$ disconnects to $\vartheta_{j}$.

\subsection{Graph-based Shortest Path}

We suppose that the tubular structures have locally lower intensities than background. The node set $\mathcal{V}$ is constructed by the prescribed tubular trajectories. These trajectories can be computed using the tubularity segmentation, with various tubular structure segmentation approaches. $\mathcal{T}_{i} \subset \Omega(i=$ $1,2, \cdots)$ denotes the trajectories and each point $x \in \mathcal{T}_{i}$ is assigned two orientations $\theta_{x}, \theta_{x}+\pi \in \mathbb{S}^{1}$.

Tubular Trajectory Extraction. We exploit the optimally oriented flux (OOF) filter [15] to segment the tubular structures and obtain the trajectories. Let $G_{\sigma}$ be a Gaussian kernel with variance $\sigma$ and let $\left\{\partial_{x_{i} x_{j}} G_{\sigma}\right\}$ be the Hessian matrix of the kernel $G_{\sigma}$. The response of the OOF filter on an image $I$ : $\Omega \rightarrow \mathbb{R}$ at a point $x$ and a scale $r \in\left[R_{\min }, R_{\max }\right]$ can be expressed as

$$
\Psi(x, r)=\left(I *\left\{\partial_{x_{i}, x_{j}} G_{\sigma}\right\}_{i, j} * \chi_{r}\right)(x),
$$

where $\chi_{r}$ is the indicator function for a disk of radius $r$ and $\left\{\partial_{x_{i}, x_{j}} G_{\sigma}\right\}_{i, j}$ is the Hessian matrix of the Gaussian kernel $G_{\sigma}$. As analyzed in [15], the confidence map, which indicates the likelihood of a point belonging to the tubular structures, 
can be computed from the eigenvalues, denoted by $\lambda_{1}(\cdot)$ and $\lambda_{2}(\cdot)$, of $\Psi(\cdot)$. Supporting that the eigenvalues $\lambda_{1}(\cdot) \leq \lambda_{2}(\cdot)$. Thus the confidence map can be denoted as

$$
\psi(x)=\min _{r \in\left[R_{\min }, R_{\max }\right]}\left\{-\frac{1}{r} \lambda_{1}(x, r), 0\right\} .
$$

Furthermore, the anisotropy features at a point $x$ located at a tubular trajectory are the two directions tangent to that trajectory. They can be estimated using the eigenvectors $\mathbf{v}_{1}\left(x, r^{*}\right)$ of the matrix $\Psi\left(x, r^{*}\right)$ corresponding to the eigenvalue $\lambda_{1}\left(x, r^{*}\right)$, where $r^{*}$ is the scale of the tubular structure should have at $x$.

The orientation $\theta_{x}$ assigned to a point $x$ of a trajectory can be estimated being such that

$$
\mathbf{n}_{\theta_{x}}=\left(\cos \theta_{x}, \sin \theta_{x}\right) \propto \mathbf{v}_{1}\left(x, r^{*}\right)
$$

where $\propto$ is the proportional operator. The image data-driven function $\mathfrak{C}$ used in Eq. (2) can be computed as

$$
\mathfrak{C}(x, \theta)=\exp \left(\alpha\left(\mathbf{n}_{\theta}^{T} \Psi\left(x, r^{*}\right) \mathbf{n}_{\theta}\right)\right),
$$

where $\alpha \in \mathbb{R}^{+}$is a constant. The value $\max \left\{-\mathbf{n}_{\theta}^{T} \Psi\left(x, r^{*}\right) \mathbf{n}_{\theta}, 0\right\}$ can be regarded as the orientation scores.

The tubular structure segmentation can be achieved by simply thresholding the confidence map $\psi$. After that the trajectories are derived by applying the morphological filters on the binary segmented tubular structures to get the skeletons. In order to obtain a set of separate trajectories, we remove all the branch points from tubular skeletons. By Eq. (8), we can obtain the orientations for each trajectory $\mathcal{T}_{i}$. Note that in this case, the trajectories are a set of connected grid points of one-point width.

Graph Construction. For the graph construction, each trajectory $\mathcal{T}_{i}$ can be regarded as a node so as to form the node set $\mathcal{V}$. For the construction of $\mathcal{E}$, we should identify the neighbouring nodes for each node $\vartheta_{i}$. This can be done by building the a tubular neighbourhood for each trajectory $\mathcal{T}_{i}$ which corresponds to the node $\vartheta_{i}$. Here we use the same method with [16] for identifying the neighbourhood region for each trajectory $\mathcal{T}_{i}$. We first prolong $\mathcal{T}_{i}$ from its two end points along the respective tangents. Then we build a regular tubular neighbourhood $M_{i}$ for extended trajectory with radius $\tau$. A trajectory $\mathcal{T}_{j}$ with $j \neq i$ is said to be connected to $\mathcal{T}_{i}$, if $M_{i} \cap \mathcal{T}_{j} \neq \emptyset$.

\subsection{Computation of the Edge Weights}

Once the construction of the edge set $\mathcal{E}$ is done, the weights $\omega_{i, j}$ for all the edges $e_{i j}$ should be identified. In this section, a new method is introduced to estimate the weights $\omega_{i, j}$ between two connected trajectories $\mathcal{T}_{i}$ and $\mathcal{T}_{j}$, i.e. two nodes $\vartheta_{i}$ and $\vartheta_{j}$, based on the FSR metric (3) and its data-driven version (2). We lift all the trajectories $\mathcal{T}_{i}$ to the orientation-lifted space to obtain the lifted trajectory $\tilde{\mathcal{T}}_{i}=\left\{\left(x, \theta_{x}\right),\left(x, \theta_{x}+\right.\right.$ $\left.\pi) ; \forall x \in \mathcal{T}_{i}\right\}$.
We first define a function $\mathcal{D}_{i, j}$ measuring the minimal curvature-penalized distance from the orientaiton-lifted trajectories $\tilde{\mathcal{T}}_{i}$ to $\tilde{\mathcal{T}}_{j}$ :

$$
\mathcal{D}_{i, j}=\inf _{\gamma(0) \in \tilde{\mathcal{T}}_{i}, \gamma(1) \in \tilde{\mathcal{T}}_{j}} \int_{0}^{1} \mathcal{F}_{\epsilon, \beta}\left(\gamma(u), \gamma^{\prime}(u)\right) d u .
$$

The distance $\mathcal{D}_{i, j}$ thus leads to a pair of points $\left(\tilde{x}^{*}, \tilde{y}^{*}\right) \in \tilde{\mathcal{T}}_{i} \times$ $\tilde{\mathcal{T}}_{j}$ such that $\mathcal{U}_{\tilde{x}^{*}}\left(\tilde{y}^{*}\right)=\mathcal{D}_{i, j}$ and a corresponding geodesic path $\mathcal{C}_{i, j}$ linking the point $\tilde{x}^{*}$ to $\tilde{y}^{*}$. It can be recovered by solving a gradient descent ordinary differential equation on the geodesic distance.

The geodesic distance $\mathcal{D}_{i, j}$ is weighted by the data-driven function $\mathfrak{C}$, which may introduce bias to the edge weights $\omega_{i, j}$. In order to overcome this shortcoming, we compute $\omega_{i, j}$ via a curvature-dependent and data-independent distance $\mathfrak{D}_{i, j}$. We reformulate the geodesic path $\mathcal{C}_{i, j}:=\left(\gamma_{i, j}, \theta_{i, j}\right)$ and the $\mathfrak{D}_{i, j}$ can be estimated by

$$
\begin{aligned}
\mathfrak{D}_{i, j} & =\int_{0}^{1} \sqrt{\left|\gamma_{i, j}^{\prime}(u)\right|^{2}+\beta_{1} \theta_{i, j}^{\prime}(u)^{2}} d u \\
& =\int_{0}^{1} \sqrt{1+\beta_{1} \kappa_{i, j}(u)^{2}}\left|\gamma_{i, j}^{\prime}(u)\right| d u
\end{aligned}
$$

where $\kappa_{i, j}$ is the curvature of $\gamma_{i, j}$ and $\beta_{1} \in \mathbb{R}^{+}$is a constant controlling the importance of the curvature. Similarly, we can obtain the distance form the distance $\mathfrak{D}_{j, i}$ by the corresponding geodesic path $\mathcal{C}_{j, i}$ with $\mathcal{C}_{j, i}(0) \in \tilde{\mathcal{T}}_{j}$ and $\mathcal{C}_{j, i}(1) \in \tilde{\mathcal{T}}_{i}$. Now we can build the weight $\omega_{i, j}$ for the edge $e_{i, j}$ :

$$
\omega_{i, j}=\min \left\{\mathfrak{D}_{i, j}, \mathfrak{D}_{j, i}\right\},
$$

yielding that $\omega_{i, j}=\omega_{j, i}$

\subsection{Tubular structure tracing}

A graph thus can be constructed and the Dijkstra's method will be applied to extract a path between two given points. The obtained rough path is the grouping of a series of ordered trajectories, which describes the tubular structures by the concatenation of these order trajectories and the respective FSR minimal paths joining them. However, the obtained curve is not able to delineate the centerline smoothly especially for a case with complicated background. We introduce the regionconstrained geodesic model to refine the final curve for tubular structure tracing. In implementation, the tubular neighboring region along the target tubular is generated by dilating the obtained rough path. Then, the FSR model tracks the real tubular structure centerlines within the neighboring region. The desired path is accurate and smooth.

\section{EXPERIMENTAL RESULTS}

We conduct the experiments with both qualitative and quantitative comparison to the anisotropic model (Aniso) [7], the 


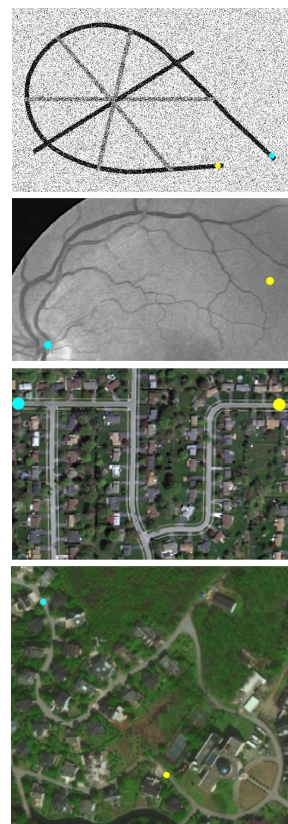

Original
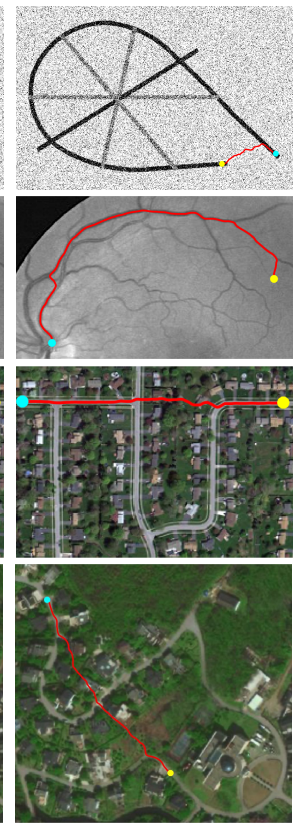

Aniso
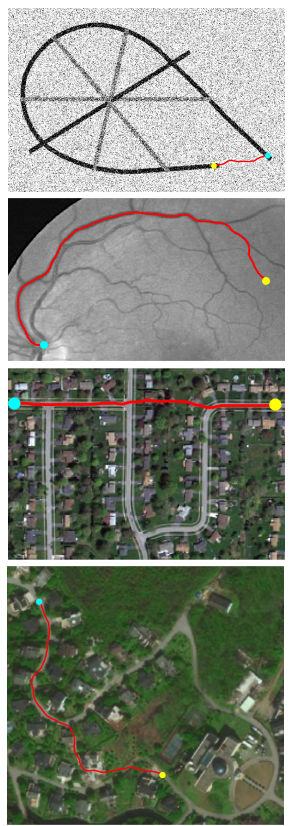

Progressive
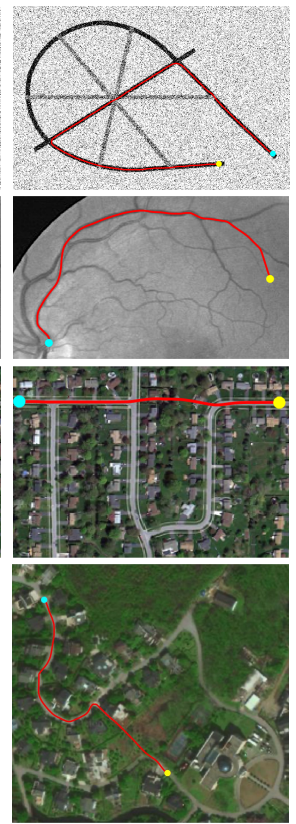

FSR
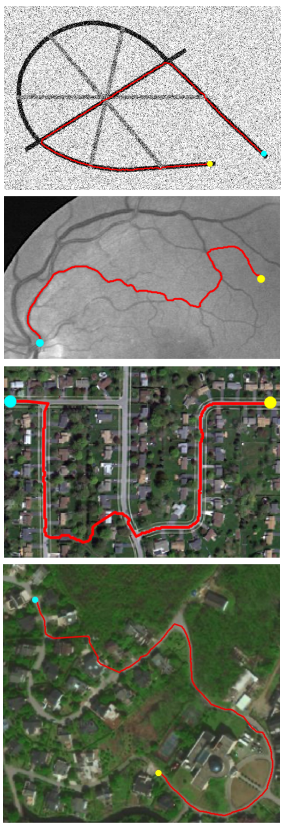

Group
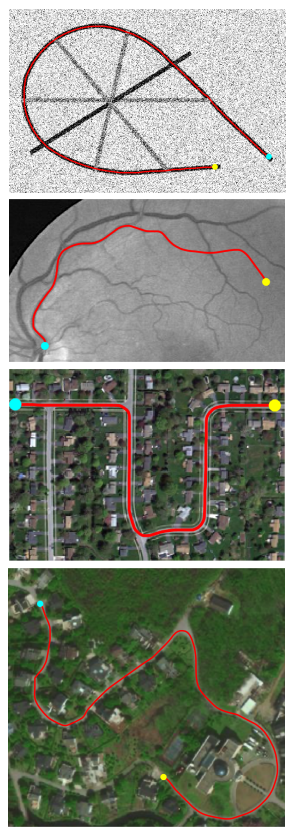

Proposed

Fig. 3: Qualitative comparison. Columns 1-6: Original images, the paths (red lines) derived from the Aniso model, the Progressive model, the FSR model, Group model and the proposed model, respectively. The cyan and yellow dots indicate the source and end points, respectively.

Table 1: Average values of $\mathcal{J}$ for evaluating the performance of the considered models in artery vessels tracing on retinal images from the DRIVE and IOSTAR datasets

\begin{tabular}{c|ccccc}
\hline Datasets & Aniso & Progressive & FSR & Group & Proposed \\
\hline DRIVE & $52.26 \%$ & $54.92 \%$ & $48.01 \%$ & $84.01 \%$ & $\mathbf{9 8 . 5 0} \%$ \\
IOSTAR & $67.09 \%$ & $74.54 \%$ & $78.71 \%$ & $85.62 \%$ & $\mathbf{9 8 . 4 3} \%$ \\
\hline
\end{tabular}

progressive model with bending constraint (Progressive) [17], the FSR model [9], the graph-based grouping method with Euclidean distance for edge weights (Group) [16] on both synthetic and real images. For fair comparison, we apply the same trajectories for Group and the proposed one.

In Fig. 3, we present experiment results for the qualitative comparison on synthetic images and real image patches involving vessel and road structures. One can observe that the results derived from the Aniso, Progressive, FSR and Group models, which are depicted in columns 1 to 4 , suffer from the short branches combination and shortcut problems. While the paths generated by the proposed, as shown in fifth column, have correctly trace the target tubular structures, thanks to the use of the curvature-penalized distance to estimate the edge weights of the graph.

The quantitative evaluation is conducted on DRIVE [18] and IOSTAR [19] retinal vessel datasets. In retinal optics images, the artery vessels appear to be weak while vein vessels are stronger. Tracing artery vessels using few user-provided points is a changeling task thus can well measure the perfor- mance of the models. We consider an accuracy score

$$
\mathcal{J}=\#|\mathbb{S} \cap \mathbb{G}| / \#|\mathbb{S}|,
$$

where $\mathbb{S}$ is the set of grid points passed through by the evaluated path, $\mathbb{G}$ denotes the region of artery vessels from the artery-vein ground truth, $\#|\mathbb{S}|$ stands for the elements involved in the set $\mathbb{S}$. Each individual artery vessel is tracked in the whole image. For most tests, we provide only 2 points, which serve as the source and end points, to extract one artery vessel and only very few cases require 3 to 5 points. We have made use of more than 1000 artery vessels sampled from two datasets. From Table. 1, the average score of the proposed model achieves more accuracy.

\section{CONCLUSION}

In this paper, we propose a new minimal path model for the delineation of tubular structure trajectories in a grouping prescribed trajectories. We introduce a natural and effective way to estimate the weights in conjunction with the curvaturepenalized geodesic distance. The experimental results prove that our model has outperformed the state-of-the-art minimal path-based tubular structure tracing models. Future work will be devoted to improve the construction of the trajectories.

\section{ACKNOWLEDGMENT}

The authors thank Dr. Jean-Marie Mirebeau from Université Paris-Sud for his fruitful discussion. 


\section{REFERENCES}

[1] E. Bekkers, R. Duits, T. Berendschot, and B. ter Haar Romeny, "A multi-orientation analysis approach to retinal vessel tracking," J. Math. Imag. Vis., vol. 49, no. 3, pp. 583-610, 2014.

[2] S. Cetin and G. Unal, "A higher-order tensor vessel tractography for segmentation of vascular structures," IEEE Trans. Med. Imag., vol. 34, no. 10, pp. 2172-2185, 2015.

[3] E. J. Bekkers, D. Chen, and J. M. Portegies, "Nilpotent approximations of sub-Riemannian distances for fast perceptual grouping of blood vessels in 2D and 3D," J. Math. Imag. Vis., vol. 60, no. 6, pp. 882-899, 2018.

[4] Y. Chen et al., "Curve-like structure extraction using minimal path propagation with backtracking," IEEE Trans. Image Process., vol. 25, no. 2, pp. 988-1003, 2016.

[5] S. Moriconi et al., "Vtrails: Inferring vessels with geodesic connectivity trees," in Proc. IPMI, 2017, pp. 672-684.

[6] L. D. Cohen and R. Kimmel, "Global minimum for active contour models: A minimal path approach," Int. J. Comput. Vis., vol. 24, no. 1, pp. 57-78, 1997.

[7] F. Benmansour and L. D. Cohen, "Tubular structure segmentation based on minimal path method and anisotropic enhancement," Int. J. Comput. Vis., vol. 92, no. 2, pp. 192-210, 2011.

[8] D. Chen, J. Zhang, and L. D. Cohen, "Minimal paths for tubular structure segmentation with coherence penalty and adaptive anisotropy," IEEE Trans. Image Process., vol. 28, no. 3, pp. 1271-1284, 2019.

[9] R. Duits, S. PL Meesters, J.-M. Mirebeau, and J. M Portegies, "Optimal paths for variants of the 2D and 3D Reeds-Shepp car with applications in image analysis," J. Math. Imag. Vis., vol. 60, no. 6, pp. 816-848, 2018.

[10] J. Ulen, P. Strandmark, and F. Kahl, "Shortest paths with higher-order regularization," IEEE Trans. Pattern Anal. Mach. Intell., vol. 37, no. 12, pp. 2588-2600, 2015.

[11] D. Chen, J.-M. Mirebeau, and L. D. Cohen, "Global minimum for a Finsler elastica minimal path approach," Int. J. Comput. Vis., vol. 122, no. 3, pp. 458-483, 2017.

[12] D. Chen, J.-M. Mirebeau, and L. D. Cohen, "A new finsler minimal path model with curvature penalization for image segmentation and closed contour detection," in Proc. CVPR, 2016, pp. 355-363.
[13] J.-M. Mirebeau, "Fast-marching methods for curvature penalized shortest paths," J. Math. Imag. Vis., vol. 60, no. 6, pp. 784-815, 2018.

[14] E. W. Dijkstra, "A note on two problems in connexion with graphs," Numer. Math., vol. 1, no. 1, pp. 269-271, 1959.

[15] M. W. Law and A. C. Chung, "Three dimensional curvilinear structure detection using optimally oriented flux," in Proc. ECCV, 2008, pp. 368-382.

[16] L. Wang et al., "Interactive retinal vessel extraction by integrating vessel tracing and graph search," in Proc. MICCAI, 2013, pp. 567-574.

[17] W. Liao et al., "Progressive minimal path method for segmentation of 2D and 3D line structures," IEEE Trans. Pattern Anal. Mach. Intell., vol. 40, no. 3, pp. 696-709, 2018.

[18] J. Staal et al., "Ridge-based vessel segmentation in color images of the retina," IEEE Trans. Med. Imag., vol. 23, no. 4, pp. 501-509, 2004.

[19] J. Zhang et al., "Robust retinal vessel segmentation via locally adaptive derivative frames in orientation scores," IEEE Trans. Med. Imag., vol. 35, no. 12, pp. 2631-2644, 2016. 